\title{
Association of Hair Concentrations of Antiretrovirals with Virologic Outcomes Among People Living with HIV in Guangxi, China
}

\author{
Quan Zhang (D) ${ }^{1,2, *}$ \\ Xiaoming $\mathrm{Li}^{\prime}$ \\ Shan Qiao' \\ Shuaifeng Liu ${ }^{3}$ \\ Zhiyong Shen ${ }^{3}$ \\ Yuejiao Zhou ${ }^{3, *}$ \\ 'South Carolina SmartState Center for \\ Healthcare Quality (CHQ), Arnold \\ School of Public Health, University of \\ South Carolina, Columbia, SC, USA; \\ ${ }^{2}$ Institute of Pedagogy and Applied \\ Psychology, School of Public \\ Administration, Hohai University, \\ Nanjing, Jiangsu, People's Republic of \\ China; ${ }^{3}$ Guangxi Zhuang Autonomous \\ Region Center for Disease Control and \\ Prevention, Nanning, Guangxi, People's \\ Republic of China
}

*These authors contributed equally to this work

\begin{abstract}
Background: Hair concentrations of antiretrovirals are an innovative and non-invasive method for measuring cumulative antiretroviral exposure and assessing long-term antiretroviral adherence. This study aimed to examine hair concentrations of antiretrovirals in relation to virologic outcomes among PLHIV in Guangxi, China.
\end{abstract}

Methods: Cross-sectional data of hair concentrations of antiretrovirals and HIV viral load were collected from 215 PLHIV in Guangxi, China. Multivariate logistic regression analyses were used to examine the association of hair concentrations of antiretrovirals with virologic outcomes.

Results: Of the 215 participants, 215, 67, and 163 PLHIV are receiving lamivudine, zidovudine, and efavirenz, respectively. Multivariate analysis revealed that hair concentrations of lamivudine [odds ratio $=16.52,95 \%$ CI $2.51-108.60, p=0.004$ ] and efavirenz [odds ratio $=14.26,95 \%$ CI 1.18-172.01, $p=0.036$ ], but not zidovudine [odds ratio $=1.77,95 \%$ CI $0.06-56.14, p=0.747$ ], were the strongest independent predictor of virologic suppression when controlling for sociodemographic and other HIV-related characteristics.

Conclusion: Hair concentrations of lamivudine and efavirenz were the strongest independent predictor of virologic suppression among Chinese PLHIV. Hair analysis of antiretrovirals may provide a non-invasive, cost-effective tool that predicts virologic suppression among PLHIV in China.

Keywords: hair concentrations, antiretroviral, viral load, people living with HIV

\section{Introduction}

Combination antiretroviral therapy (cART) is the primary modality for treating and preventing HIV infection and can substantially reduce HIV-related morbidity, mortality, and transmission. ${ }^{1,2}$ Optimal cART adherence is a critical determinant for adequate antiretroviral exposure, which has been vital to virologic suppression and improved clinical outcomes among people living with HIV (PLHIV). ${ }^{3-5}$ Given the pharmacological relationship between cART adherence and antiretroviral exposure, analysis of antiretroviral concentrations in biological samples has been used as an objective method for measuring antiretroviral exposure and assessing antiretroviral adherence. Pharmacologic markers of antiretroviral exposure and antiretroviral adherence often involve measurement of antiretroviral concentrations in biological samples, such as plasma, ${ }^{6,7}$ urine, ${ }^{8}$ peripheral blood mononuclear cells (PMBC), dried blood spots (DBS), ${ }^{10,11}$ and hair. ${ }^{12,13}$ Among those metrics, plasma and urine levels can only measure real-time or short-term antiretroviral exposure and 
antiretroviral adherence (eg, hours to days). In addition, plasma and urine antiretroviral levels are susceptible to day-to-day variation. ${ }^{4,14}$ Until now, DBS levels can measure cumulative exposure to several antiretrovirals that are activated intracellularly ${ }^{4,15}$ and longer-term antiretroviral adherence (eg, days to weeks). Alternatively, hair levels can measure cumulative exposure to all antiretrovirals ${ }^{4,16,17}$ and long-term antiretroviral adherence (eg, weeks to months) because hair levels reflect antiretroviral uptake from the systemic circulation over weeks to months. In addition, hair collection is non-invasive and requires minimal training, and hair specimens can be stored and shipped at room temperature without biohazardous precautions. ${ }^{18,19}$ All of these advantages make hair concentrations of antiretrovirals appealing as an innovative method for measuring cumulative antiretroviral exposure and assessing long-term antiretroviral adherence.

Presently, there are more than 25 antiretrovirals in multiple classes approved by US Food and Drug Administration for HIV treatment, such as nucleoside reverse transcriptase inhibitors (NRTI), non-nucleoside reverse transcriptase inhibitors (NNRTI), protease inhibitors (PI), and integrase strand transfer inhibitor (INSTI, eg, raltegravir). ${ }^{20}$ Worldwide, recommended cART is consisting of two or more classes of antiretrovirals. For example, currently, WHO recommends one INSTI (eg, dolutegravir) and two NRTIs [eg, lamivudine (3TC), zidovudine (AZT), tenofovir (TFV)] as the first-line treatment, one NNRTI [eg, efavirenz (EFV), nevirapine (NVP)] and two NRTIs as alternative first-line treatment, and one PI [eg, atazanavir (ATV) and lopinavir/ritonavir (LPV/RTV)] and two NRTIs as the second-line treatment. As evidenced by a comprehensive review, ${ }^{4}$ an ever-increasing body of literature has shown that more than ten hair concentrations of antiretrovirals were employed as biomarkers of cumulative antiretroviral exposure and/or long-term antiretroviral adherence to predict virologic outcomes in Africa, North America, and Europe settings. Although China rolled out its free cART project for HIV treatment in 2004, and access to first- or second-line cART has been scaled up across the country, limited data are available on the association of hair concentrations of antiretrovirals with virologic outcomes among Chinese PLHIV. Furthermore, the simultaneous determination of hair concentrations of antiretrovirals in multiple classes is technically possible. ${ }^{21-24}$ However, major of the previous studies only investigated the association of hair concentrations of a single antiretroviral ${ }^{13,25-33}$ or antiretrovirals in a single class ${ }^{34}$ with virologic outcomes. Limited data are available on the association of hair concentrations of antiretrovirals in multiple classes with virologic outcomes.

Accordingly, we present this analysis of hair concentrations of three antiretrovirals (3TC, AZT, and EFV) in two classes (NRTI and NNRTI) in relation to virologic outcomes among Chinese PLHIV.

\section{Materials and Methods}

\section{Participants}

The participants of this study were recruited from Wave 3 of the HIV disclosure project, which is a longitudinal study that aims to explore the mechanism underlying the linkage between HIV disclosure and clinical outcomes in Guangxi, China. ${ }^{35-37}$ With the Guangxi Center for Disease Prevention and Control (Guangxi CDC) 's assistance and collaboration, we randomly selected 10 clinic sites with the largest number of HIV/AIDS cases from 17 cities and 75 counties in Guangxi. The recruitment procedure, including inclusion and exclusion criteria, was described in the previous studies. ${ }^{35}$ Briefly, medical staff or HIV case managers at the study sites referred potential participants to the research team members. Research team members screened PLHIV for eligibility, discussed the benefits and risks of the study, and invited them to participate. Research team members were local CDC staff or healthcare workers in the HIV clinics who had received intensive training on research ethics and interview skills with PLHIV before the field data and hair specimen collection. Finally, a total of 446 PLHIV participated in Wave 1 of the HIV disclosure project, and 428 PLHIV participated in Wave 3 of the HIV disclosure project.

This study followed the Declaration of Helsinki and was approved by ethical review boards of the University of South Carolina (Columbia, USA) and the Guangxi Zhuang Autonomous Region Center for Disease Prevention and Control (Nanning, China). The written informed consent also has been obtained from the participants.

\section{Hair Sample Collection and Assay}

Hair samples were cut from the posterior vertex region as close as possible to the scalp following a standard protocol $^{38,39}$ after participants finished the survey. The hair sample (about $1 \mathrm{~cm}$ of the proximal section) was rinsed with methanol and dried under a blow of pure nitrogen gas. Then, hair sample was chopped to $1-2 \mathrm{~mm}$ 
length segments with scissors, and $10 \mathrm{mg}$ weighed, processed, and analyzed using high-performance liquid chromatography (Agilent 1200 HPLC system, Agilent, Waldbronn, Germany) and tandem mass spectrometry (ABI 3200Qtrap, ABI, Foster City, CA, USA) (LC-MS /MS) as described in previous studies. ${ }^{21,24,40}$ Briefly, following the 2013 FDA guidelines, antiretrovirals in the cut hair samples were extracted with methanol and internal standard in a $37^{\circ} \mathrm{C}$ shaking water bath overnight $(>16$ hours) and then analyzed by an LC-MS/MS. Standard curves were linear in the range of 6-40,000 pg for 3TC, $10-40,000 \mathrm{pg}$ for AZT, and 12-40,000 pg for EFV with good linearity and reproducibility. The relative error (\%) and precision [coefficients of variation] for spiked quality control hair samples at low, medium, and high concentrations were all $<13 \%$. The recoveries at low, medium, and high concentrations were all $\geq 91.1 \%$. The lower limit of quantitation was $6 \mathrm{pg} / \mathrm{mg}, 10 \mathrm{pg} / \mathrm{mg}$, and $12 \mathrm{pg} / \mathrm{mg}$ for 3TC, AZT, and EFV.

\section{Sociodemographic and HIV-Related Characteristics}

Participants provided information on their sociodemographic characteristics, including age (years), gender (male vs female), ethnicity (Han vs non-Han), marital status (married vs other), education level ( $>9$ years vs $\leq 9$ years), employment status (employed vs unemployed), monthly household income level $(\geq 2000$ Chinese Yuan vs $<2000$ Chinese Yuan).

The participants' HIV viral load and other HIV-related characteristics at the time of hair collection, including date of HIV diagnosis, current cART status, date of starting current cART, and CD4 count, were extracted from their clinical records. Given that the "Undetectable $=$ Untransmittable" initiative defined undetectable viral load as viral load less than 200 copies/mL that cannot sexually transmit HIV to others, ${ }^{41}$ we defined virologic suppression as viral load less than 200 copies/mL, and virologic failure as viral load equal or more than 200 copies $/ \mathrm{mL}$. Years of HIV diagnosis and current cART referred to the time period from the initial date of confirmed HIV diagnosis and the start date of current cART to the date of hair collection, respectively. The cART status was category into first-line and second-line cART. CD4count was dichotomized as $\geq 350$ cells $/ \mathrm{mm}^{3}$ vs $<350$ cells $/ \mathrm{mm}^{3}$ because free cART was offered to PLHIV, whose CD4 count was less than 350 cells $/ \mathrm{mm}^{3}$.
Self-reported adherence was determined by asking participants to indicate how often they took their antiretrovirals as prescribed in the past month. Response options ranged from 1 (100\% of the time) to 5 (I have not taken any of my prescribed medications). This measure has been found to predict CD4+ T cell counts and viral load as well as, or better than, other measures of self-reported adherence. $^{42}$ Given that self-reported data are generally skewed and overestimated, we dichotomized self-reported adherence to $100 \%$ and less than $100 \%{ }^{36}$

\section{Statistical Analysis}

Of 428 PLHIV, 213 were excluded from the final analysis because they had not yet started cART $(n=13)$ or stopped cART $(n=2)$ or unavailable HIV viral load $(n=198)$, leaving an effective sample of 215 participants for analysis.

Descriptive statistics were used to summarize hair concentrations of antiretrovirals, sociodemographic, and HIVrelated characteristics. Categorized data were expressed as number (n) and percentage (\%). A one-sample ShapiroWilks test was used to examine normally distributed data. Non-normally distributed data were expressed as the median and interquartile range (IQR), and normally distributed data were expressed as mean (M) and standard deviation (SD). Chi-square test (for categorical variables), $t$-test (for normally distributed continuous variables), and MannWhitney $U$-test (for non-normally distributed continuous variables) were used to compare differences between the virologic suppression and virologic failure groups in terms of the sociodemographic, hair concentrations of antiretrovirals, and other HIV-related characteristics. Spearman correlation analysis was performed to examine the association among hair concentrations of antiretrovirals and the association of hair concentrations of antiretrovirals with sociodemographic and other HIV-related variables.

Multivariate linear regression analysis was used to check for collinearity, and all variables showed good tolerance $(>0.1)$ and variance inflation factors $(<10)$. Logistic regression analysis was used to estimate the association of hair concentrations of antiretrovirals (3TC, AZT, and EFV separately) with the dichotomous outcome of virologic response. Hair concentrations of 3TC, AZT, and EFV tertiles were calculated, and the second and third tertiles were contrasted with the first tertile as the reference category. The univariate logistic regression model was used to examine those associations. To fully control the potential impact of sociodemographics and other HIV-related variables on virologic response, the multivariate logistic regression model with 
the enter method was used to examine those associations adjusting sociodemographics and other HIV-related variables. Crude odds ratios (cORs) and their $95 \%$ confidence interval $(95 \% \mathrm{CI})$ were reported for univariate analysis, and adjusted odds ratios (aORs) and their 95\% CI were reported for multivariate analysis. All data analyses were performed using SPSS 26.0 (SPSS Inc., Chicago, IL).

\section{Results}

Of the 215 PLHIV with a mean (SD) age of 41 (8) years (Table 1), 68.4\% were male, 55.7\% were Han ethnicity,
$78.6 \%$ were married, $86.5 \%$ were employed, $86 \%$ were receiving the first-line cART, $84.2 \%$ were reporting $100 \%$ cART adherence, $52.6 \%$ were achieving CD4 count- $\geq 350$ cells $/ \mathrm{mm}^{3}$, and $91.6 \%$ were achieving virologic suppression. Most of the sample had low levels of education and income, with $96.1 \%$ reporting not complete junior high school and 54\% reporting a monthly household income of less than 2000 Chinese Yuan (or approximately US\$300 during the time of the study). The median years of HIV diagnosis and current cART were 2.8 years and 2.7 years. Significant differences between virologic suppression and

Table I Sociodemographic and HIV-Related Characteristics of Participants

\begin{tabular}{|c|c|c|c|c|}
\hline Characteristics & $\begin{array}{l}\text { Total } \\
n=215(100 \%)\end{array}$ & $\begin{array}{l}\text { Virologic Suppression } \\
n=197(91.6 \%)\end{array}$ & $\begin{array}{l}\text { Virologic Failure } \\
n=18(8.4 \%)\end{array}$ & p-value \\
\hline Age, years & $43.13 \pm 8.31$ & $43.44 \pm 11.71$ & $43.10 \pm 7.97$ & 0.865 \\
\hline \multicolumn{5}{|l|}{ Gender } \\
\hline Male & 147 (68.4\%) & 137 (69.5\%) & $10(55.6 \%)$ & 0.222 \\
\hline Female & $68(31.6 \%)$ & $60(30.5 \%)$ & 8 (44.4\%) & \\
\hline \multicolumn{5}{|l|}{ Ethnicity } \\
\hline Han & $122(56.7 \%)$ & $113(57.4 \%)$ & $9(50.0 \%)$ & 0.546 \\
\hline No-Han & 93 (44.3\%) & 84 (42.6\%) & $9(50.0 \%)$ & \\
\hline \multicolumn{5}{|l|}{ Marital status } \\
\hline Married & $169(78.6 \%)$ & $157(79.7 \%)$ & $12(66.7 \%)$ & 0.197 \\
\hline Others & $46(21.4 \%)$ & $40(20.3 \%)$ & $6(33.3 \%)$ & \\
\hline \multicolumn{5}{|l|}{ Education levels } \\
\hline$>9$ years & $18(8.4 \%)$ & $18(9.1 \%)$ & $0(0 \%)$ & 0.180 \\
\hline$\leq 9$ years & 197 (91.6\%) & $179(90.9 \%)$ & $18(100 \%)$ & \\
\hline \multicolumn{5}{|l|}{ Employment status } \\
\hline Employed & 186 (86.5\%) & I 72 (87.3\%) & $14(77.8 \%)$ & 0.257 \\
\hline Unemployed & 29 (13.5\%) & 25 (12.7\%) & $4(22.2)$ & \\
\hline \multicolumn{5}{|l|}{ Monthly income levels } \\
\hline$\geq 2000$ Chinese Yuan & $99(46.0 \%)$ & $92(46.7 \%)$ & 7 (38.9\%) & 0.524 \\
\hline$<2000$ Chinese Yuan & $116(54.0 \%)$ & $105(53.3 \%)$ & $11(61.6 \%)$ & \\
\hline Years of HIV diagnosis, years & $2.8(2.3-3.4)$ & $2.8(2.3-3.4)$ & $2.5(1.9-3.3)$ & 0.228 \\
\hline \multicolumn{5}{|l|}{ Current cART status } \\
\hline First-line cART & I85 (86.0\%) & I 73 (87.8\%) & $12(66.7 \%)$ & 0.013 \\
\hline Second-line cART & $30(14.0 \%)$ & $24(12.2 \%)$ & $6(33.3 \%)$ & \\
\hline Years of current cART, years & $2.7(2.3-3.4)$ & $2.8(2.3-3.4)$ & $2.5(1.9-3.2)$ & 0.249 \\
\hline \multicolumn{5}{|l|}{ CD4 count-, cells $/ \mathrm{mm}^{3}$} \\
\hline$<350$ & $102(47.4 \%)$ & $88(44.7 \%)$ & $14(77.8 \%)$ & 0.007 \\
\hline$\geq 350$ & $113(52.6 \%)$ & $109(55.3 \%)$ & $4(22.4 \%)$ & \\
\hline \multicolumn{5}{|l|}{ Self-reported adherence } \\
\hline $100 \%$ & I8I (84.2\%) & 167 (93.2\%) & $14(77.8 \%)$ & 0.436 \\
\hline Less than $100 \%$ & $34(15.8 \%)$ & 30 (I5.2\%) & $4(22.2 \%)$ & \\
\hline
\end{tabular}

Abbreviation: cART, combination antiretroviral therapy. 
Table 2 Correlation Between Hair Concentrations of Antiretrovirals and Sociodemographic and Other Clinical Characteristics

\begin{tabular}{|c|c|c|c|c|c|c|}
\hline \multirow[t]{2}{*}{ Characteristics } & \multicolumn{2}{|c|}{$\begin{array}{l}\text { 3TC Concentrations } \\
\mathrm{N}=2 \text { I } 5\end{array}$} & \multicolumn{2}{|c|}{$\begin{array}{l}\text { AZT Concentrations } \\
\mathrm{N}=67\end{array}$} & \multicolumn{2}{|c|}{$\begin{array}{l}\text { EFV Concentrations } \\
N=163\end{array}$} \\
\hline & $r$ & p-value & $r$ & p-value & $r$ & p-value \\
\hline Age & 0.100 & 0.142 & 0.026 & 0.836 & 0.005 & 0.947 \\
\hline Gender & 0.114 & 0.097 & 0.188 & 0.127 & 0.127 & 0.107 \\
\hline Ethnicity & -0.034 & 0.662 & 0.123 & 0.321 & 0.083 & 0.293 \\
\hline Marital status & -0.050 & 0.468 & 0.022 & 0.863 & -0.089 & 0.259 \\
\hline Education levels & 0.120 & 0.080 & 0.043 & 0.730 & -0.023 & 0.770 \\
\hline Employment status & -0.170 & 0.013 & -0.130 & 0.294 & -0.028 & 0.724 \\
\hline Monthly income levels & 0.112 & 0.102 & 0.193 & 0.024 & 0.031 & 0.693 \\
\hline Years of HIV diagnosis & 0.105 & 0.125 & -0.097 & 0.435 & -0.126 & 0.108 \\
\hline Current cART satus & 0.127 & 0.063 & -0.245 & 0.045 & - & - \\
\hline Years of current cART & 0.114 & 0.096 & -0.097 & 0.435 & -0.090 & 0.252 \\
\hline Self-reported adherence & 0.181 & 0.008 & 0.340 & 0.005 & 0.149 & 0.057 \\
\hline CD4 count & -0.016 & 0.811 & 0.240 & 0.097 & -0.104 & 0.186 \\
\hline
\end{tabular}

Abbreviations: cART, combination antiretroviral therapy; 3TC, lamivudine; AZT, zidovudine; EFV, efavirenz.

virologic failure groups were only found in current cART status $(p=0.013)$ and CD4 count $(p=0.007)$.

As shown in Table 2, marginal or significant correlation was found between hair 3TC concentrations and gender $(p=0.097)$, education levels $(p=0.080)$, employment status $(p=0.013)$, current cART status $(p=0.063)$, years of current cART $(p=0.096)$ and self-reported adherence $(p=0.008)$, between hair AZT concentrations and monthly income levels ( $p=0.024)$, current cART status $(p=0.045)$, self-reported adherence $(p=0.005)$ and CD4 count $(p=$ 0.097), and between hair EFV concentrations and selfreported adherence $(p=0.057)$. In addition, positive correlations were found between hair 3TC concentrations and hair AZT concentrations ( $\mathrm{n}=68, r=0.465, p<0.001$ ), between hair 3TC concentrations and hair EFV concentrations $(\mathrm{n}=163, r=0.414, p<0.001)$, and between hair AZT concentrations and hair EFV concentrations ( $\mathrm{n}=38, r=0.597, p<0.001)$.

As shown in Table 3, of the 215 participants, 215, 67, and 163 PLHIV are receiving 3TC, AZT, and EFV, and 197, 60, and 153 PLHIV are achieving virologic suppression, respectively. PLHIV with virologic suppression had significant higher hair concentrations of 3TC, AZT, and EFV than those with virologic failure $(p=0.008, p=$ 0.016 , and $p=0.030$, respectively). The median and interquartile range (IQR) of hair concentrations of 3TC, AZT, and EFV among those with virological suppression was 476.55 (315.33-695.38) pg/mg, 289.48 (166.35-471.21) $\mathrm{pg} / \mathrm{mg}$, and 4814.74 (3472.07-7111.86) $\mathrm{pg} / \mathrm{mg}$, respectively. The value of $315.33 \mathrm{pg} / \mathrm{mg}, 166.35 \mathrm{pg} / \mathrm{mg}$, and

Table 3 Hair Concentrations of Antiretrovirals by the Virologic Outcome

\begin{tabular}{|l|l|l|l|l|l|}
\hline \multirow{2}{*}{ Antiretrovirals } & \multicolumn{2}{|l|}{ Virologic Suppression } & \multicolumn{2}{l|}{ Virologic Failure } & p-value \\
\cline { 2 - 6 } & $\mathbf{n}$ & Median (IQR), pg/mg & $\mathbf{n}$ & Median (IQR), pg/mg \\
\hline 3TC & 197 & $476.55(315.33-695.38)$ & 18 & $247.42(36.66-454.67)$ & 0.008 \\
\hline AZT & 60 & $289.48(166.35-471.21)$ & 7 & $10.00(10.00-185.98)$ & 0.016 \\
\hline EFV & 153 & $4814.74(3472.07-7111.86)$ & 10 & $3262.33(53.18-5252.53)$ & 0.030 \\
\hline
\end{tabular}

Abbreviations: IQR, interquartile range; 3TC, lamivudine; AZT, zidovudine; EFV, efavirenz. 
Table 4 Logistic Regression Results Predicting Virologic Suppression Based on Hair Concentrations of Antiretrovirals

\begin{tabular}{|c|c|c|c|c|c|}
\hline & \multirow[b]{2}{*}{ n (\%) } & \multicolumn{2}{|c|}{ Univariate } & \multicolumn{2}{|c|}{ Multivariate } \\
\hline & & cORs $(95 \% \mathrm{Cl})$ & $\mathbf{p}$ & aORs $(95 \% \mathrm{Cl})$ & $p$ \\
\hline \multicolumn{6}{|l|}{3 TC $(n=2 \mid 5)$} \\
\hline Lowest tertile & $60(84.5 \%)$ & 1.00 (ref) & - & 1.00 (ref) & - \\
\hline Middle tertile & 67 (94.4\%) & $3.07(0.93-10.16)$ & 0.066 & $5.65(1.21-26.34)$ & 0.028 \\
\hline Highest tertile & 70 (95.9\%) & $4.28(1.14-16.05)$ & 0.031 & $16.52(2.5 I-108.60)$ & 0.004 \\
\hline \multicolumn{6}{|l|}{ AZT $(n=67)$} \\
\hline Lowest tertile & 17 (77.3\%) & 1.00 (ref) & - & & \\
\hline Middle tertile & 21 (95.5\%) & $6.18(0.66-58.03)$ & 0.111 & $1.06(0.02-47.85)$ & 0.976 \\
\hline Highest tertile & 22 (95.7\%) & $6.47(0.69-60.68)$ & 0.102 & $1.77(0.06-56.14)$ & 0.747 \\
\hline \multicolumn{6}{|l|}{$\operatorname{EFV}(n=163)$} \\
\hline Lowest tertile & 49 (90.7\%) & 1.00 (ref) & - & & \\
\hline Middle tertile & $50(92.6 \%)$ & $1.28(0.32-5.02)$ & 0.728 & $1.72(0.34-8.78)$ & $0.5 \mathrm{II}$ \\
\hline Highest tertile & 54 (98.2\%) & $5.5 \mathrm{I}(0.62-48.82)$ & 0.125 & $14.26(1.18-172.01)$ & 0.036 \\
\hline
\end{tabular}

Abbreviations: $\mathrm{Cl}$, confidence interval; cOR, crude odds ratio; aOR, adjusted odds ratio; 3TC, lamivudine; AZT, zidovudine; $\mathrm{EFV}$, efavirenz.

$3472.07 \mathrm{pg} / \mathrm{mg}$ (lower IQR for those with virological suppression) defined a cut-off below which most participants experienced virological failure.

The results of logistic regression models of virologic suppression are presented in Table 4 . The cORs were 4.28 [95\% CI 1.14-16.05, $p=0.031$ ], 6.47 [95\% CI 0.69-60.68, $\mathrm{p}=0.102]$, and 5.51 [95\% CI $0.62-48.82, \mathrm{p}=0.125]$ for those with hair concentrations of 3TC, AZT, and EFV in the highest tertile compared to the lowest tertile in univariate analysis. The aORs were 16.52 [95\% CI 2.51-108.60, $\mathrm{p}=0.004$ ], 1.77 [95\% CI 0.06-56.14, $\mathrm{p}=0.747$ ], and 14.26 [95\% CI 1.18-172.01, $\mathrm{p}=0.036]$ for those with hair concentrations of 3TC, AZT, and EFV in the highest quartile compared to the lowest quartile when controlling for sociodemographic and other HIV-related characteristics.

\section{Discussion}

Our study is the first to examine the association of hair concentrations of various antiretrovirals with virologic outcomes in a cohort of Chinese PLHIV on cART. We show that, while hair concentrations of 3TC, AZT, and EFV were significantly higher in PLHIV with virologic suppression than those with virologic failure, hair concentrations of 3TC and EFV, but not AZT, are the strongest independent predictor of virologic suppression when controlling for sociodemographic, and other HIV-related characteristics. In addition, we found positive correlations among hair concentrations of antiretrovirals and between self-reported adherence and hair concentrations of antiretrovirals.
Our results regarding PLHIV with virologic suppression had higher hair concentrations of 3TC, AZT, and EFV than those with virologic failure were in line with previous studies employing hair concentrations of $3 \mathrm{TC},{ }^{13} \mathrm{EFV},{ }^{12,43}$ $\mathrm{TFV}^{33}{ }^{33} \mathrm{NVP},{ }^{12} \mathrm{LPV}^{12,29,31,34,43} \mathrm{RTV}^{12,29,34} \mathrm{ATV}^{34,44}$ and indinavir. $^{25-27}$ Interestingly, our study revealed that hair concentrations of $3 \mathrm{TC}$ and EFV, but not AZT, are the strongest independent predictor of virologic suppression. Our findings of hair concentrations of 3TC and EFV added evidence to existing studies that reported that hair concentrations of antiretrovirals (eg, $\mathrm{TFV}^{33} \mathrm{EFV}^{43,45} \mathrm{NVP},{ }^{30}$ $\mathrm{LPV}^{31,32,34} \mathrm{ATV}^{28,34}$ ) were the strongest independent predictor of virologic suppression. Regarding the nonsignificant finding of hair AZT concentrations, the small sample size and lower hair AZT concentrations could contribute to this finding. As seen in Table 3, among 215 PLHIV on cART, only 67 PLHIV were receiving AZT-based cART, and hair AZT concentrations were much lower than hair concentrations of 3TC and EFV.

We found a positive correlation between self-reported adherence and hair concentrations of 3TC, AZT, and EFV, which was partly in line with previous studies by using hair concentrations of antiretrovirals (eg, $\mathrm{ATV}^{28}$ and $\mathrm{TFV}^{36}$ ). This finding might also indicate that our study's adherence measure could be better detection of cART adherence. ${ }^{42}$ In addition, we found positive correlations among hair concentrations of 3TC, AZT, and EFV, which might indicate a significant drug-drug interaction among those antiretrovirals. Moreover, we found that hair 3TC concentrations were easily affected by sociodemographic 
and HIV-related characteristics than hair concentrations of AZT and EFV did (Table 2), which added not only the understanding of the factors related to hair concentrations of antiretrovirals, ${ }^{19,37}$ but also the potential reason that hair EFV concentrations had higher effect than hair 3TC concentrations, in addition to hair EFV concentrations are much higher than hair 3TC concentrations.

Our study has several limitations. First, the current study was based on cross-sectional data, which prevents making causal inferences. Second, the current study only employed hair concentrations of three antiretrovirals. Future studies should examine some other commonly used free antiretrovirals (eg, TFV, NVP, and LPV/RTV) in a large sample size with a more innovative methodology (eg, machine learning). Third, we employed the lower IQR of hair concentrations of antiretrovirals for those with virologic- suppression as the recommended cut-offs for viral failures based on the Chawana et al. ${ }^{44}$ Future studies should determine such cut-offs using pharmacokinetic modeling. ${ }^{46}$ Fourth, because all participants were from Guangxi, China, these findings may not be generalizable to PLHIV in other settings. Future research should employ both Chinese PLHIV and PLHIV in other countries to examine the association between hair concentrations of antiretrovirals with virologic outcomes. Finally, data were not available in the current study on some factors (eg, illegal drugs use) that might influence virologic outcomes or hair concentrations of antiretrovirals. ${ }^{19}$ Those factors should be considered in future research.

\section{Conclusion}

In summary, our findings indicated that hair concentrations of antiretrovirals were significantly associated with virologic outcomes among Chinese PLHIV. Hair analysis of antiretrovirals may provide a non-invasive, cost-effective tool that predicts virologic suppression among Chinese PLHIV.

\section{Acknowledgments}

The authors thank the study participants and data collectors for their collaboration during the data collection. Quan Zhang and Yuejiao Zhou share co-first authorship.

\section{Funding}

This study was supported by the National Institutes of Health (NIH) Research Grant [Grant numbers R01HD074221, R21AI122919].

\section{Disclosure}

The authors report no conflicts of interest in this work.

\section{References}

1. Saag MS, Benson CA, Gandhi RT, et al. Antiretroviral drugs for treatment and prevention of HIV infection in adults: 2018 recommendations of the international antiviral society-USA panel. JAMA. 2018;320(4):379-396. doi:10.1001/jama.2018.8431

2. Altice F, Evuarherhe O, Shina S, Carter G, Beaubrun AC. Adherence to HIV treatment regimens: systematic literature review and meta-analysis. Patient Prefer Adherence. 2019;13:475-490. doi:10.2147/PPA.S192735

3. Robbins RN, Spector AY, Mellins CA, Remien RH. Optimizing ART adherence: update for HIV treatment and prevention. Curr HIV/AIDS Rep. 2014;11(4):423-433. doi:10.1007/s11904-014-0229-5

4. Zhang Q, Qiao S, Yang X, Li X. Antiretroviral concentration in hair as a measure for antiretroviral medication adherence: a systematic review of global literature. AIDS Behav. 2020;24(1):311-330. doi:10.1007/s10461-019-02460-5

5. Saberi P, Ming K, Legnitto D, Neilands TB, Gandhi M, Johnson MO Novel methods to estimate antiretroviral adherence: protocol for a longitudinal study. Patient Prefer Adherence. 2018;12:1033-1042. doi:10.2147/PPA.S166380

6. Marzolini CTA, Decosterd LA, Greub G, Biollaz J, Buclin T. Efavirenz plasma levels can predict treatment failure and central nervous system side effects in HIV-1-infected patients. AIDS. 2001;15(1):71-75. doi:10.1097/00002030-200101050-00011

7. Mwasakifwa GE, Moore C, Carey D, et al. Relationship between untimed plasma lopinavir concentrations and virological outcome on second-line antiretroviral therapy. AIDS. 2018;32(3):357-361. doi:10.1097/QAD.0000000000001688

8. Gandhi M, Bacchetti P, Spinelli MA, et al. Brief report: validation of a urine tenofovir immunoassay for adherence monitoring to PrEP and ART and establishing the cutoff for a point-of-care test. $J$ Acquir Immune Defic Syndr. 2019;81(1):72-77. doi:10.1097/QAI.0000000000001971

9. Baxi SM, Liu A, Bacchetti P, et al. Comparing the novel method of assessing PrEP adherence/exposure using hair samples to other pharmacologic and traditional measures. J Acquir Immune Defic Syndr. 2015;68(1):13-20. doi:10.1097/QAI.0000000000000386

10. Frasca K, Morrow M, Coyle RP, et al. Emtricitabine triphosphate in dried blood spots is a predictor of viral suppression in HIV infection and reflects short-term adherence to antiretroviral therapy. J Antimicrob Chemother. 2019;74(5):1395-1401. doi:10.1093/jac/ dky559

11. Morrow M, MaWhinney S, Coyle RP, et al. Predictive value of tenofovir diphosphate in dried blood spots for future viremia in persons living with HIV. J Infect Dis. 2019;220(4):635-642. doi:10.1093/infdis/jiz144

12. Tabb ZJ, Mmbaga BT, Gandhi M, et al. Antiretroviral drug concentrations in hair are associated with virologic outcomes among young people living with HIV in Tanzania. AIDS. 2018;32(9):1115-1123. doi:10.1097/QAD.0000000000001788

13. Yan J, Liu J, Su B, et al. Lamivudine concentration in hair and prediction of virologic failure and drug resistance among HIV patients receiving free ART in China. PLoS One. 2016;11(4): e0154421. doi:10.1371/journal.pone.0154421

14. Nettles RE, Kieffer TL, Parsons T, et al. Marked intraindividual variability in antiretroviral concentrations may limit the utility of therapeutic drug monitoring. Clin Infect Dis. 2006;42(8):1189-1196. doi: $10.1086 / 501458$

15. Gandhi M, Gandhi RT, Stefanescu A, et al. Cumulative antiretroviral exposure measured in hair is not associated with measures of HIV persistence or inflammation among individuals on suppressive ART. J Infect Dis. 2018;218(2):234-238. doi:10.1093/infdis/jiy011 
16. Castillo-Mancilla JR, Haberer JE. Adherence measurements in HIV: new advancements in pharmacologic methods and real-time monitoring. Curr HIV/AIDS Rep. 2018;15(1):49-59. doi:10.1007/ s11904-018-0377-0

17. Gandhi M, Greenblatt RM. Hair it is: the long and short of monitoring antiretroviral treatment. Ann Intern Med. 2002;137(8):696-697. doi:10.7326/0003-4819-137-8-200210150-00016

18. Hickey MD, Salmen CR, Tessler RA, et al. Antiretroviral concentrations in small hair samples as a feasible marker of adherence in rural Kenya. $J$ Acquir Immune Defic Syndr. 2014;66(3):311-315. doi:10.1097/QAI.0000000000000154

19. Zhang Q, Li X, Qiao S, Yang X. Factors related to hair antiretroviral concentration: a systematic review of global literature. AIDS Rev. 2020;22(1):25-33. doi:10.24875/AIDSRev.19000122

20. De Clercq E. Anti-HIV drugs: 25 compounds approved within 25 years after the discovery of HIV. Int J Antimicrob Agents. 2009;33 (4):307-320. doi:10.1016/j.ijantimicag.2008.10.010

21. Chu L, Wu Y, Duan C, et al. Simultaneous quantitation of zidovudine, efavirenz, lopinavir and ritonavir in human hair by liquid chromatography-atmospheric pressure chemical ionization-tandem mass spectrometry. J Chromatogr B Analyt Technol Biomed Life Sci. 2018;1097-1098:54-63. doi:10.1016/j.jchromb.2018.08.031

22. Huang Y, Gandhi M, Greenblatt RM, Gee W, Lin ET, Messenkoff N. Sensitive analysis of anti-HIV drugs, efavirenz, lopinavir and ritonavir, in human hair by liquid chromatography coupled with tandem mass spectrometry. Rapid Commun Mass Spectrom. 2008;22 (21):3401-3409. doi:10.1002/rcm.3750

23. Shah SA, Mullin R, Jones G, et al. Simultaneous analysis of antiretroviral drugs abacavir and tenofovir in human hair by liquid chromatography-tandem mass spectrometry. J Pharm Biomed Anal. 2013;74:308-313. doi:10.1016/j.jpba.2012.10.023

24. Wu Y, Yang J, Duan C, et al. Simultaneous determination of antiretroviral drugs in human hair with liquid chromatography-electrospray ionization-tandem mass spectrometry. $J$ Chromatogr B Analyt Technol Biomed Life Sci. 2018;1083:209-221. doi:10.1016/j.jchromb.2018.03.021

25. Bernard L, Peytavin G, Vuagnat A, de Truchis P, Perronne C. Indinavir concentrations in hair from patients receiving highly active antiretroviral therapy. The Lancet. 1998;352(9142):1757-1758. doi:10.1016/S0140-6736(05)79831-7

26. Bernard L, Vuagnat A, Peytavin G, et al. Relationship between levels of indinavir in hair and virologic response to highly active antiretroviral therapy. Ann Intern Med. 2002;137(8):656-659. doi:10.7326/ 0003-4819-137-8-200210150-00009

27. Duval X, Peytavin G, Breton G, et al. Hair versus plasma concentrations as indicator of indinavir exposure in HIV-1-infected patients treated with indinavir/ritonavir combination. AIDS. 2007;21 (1):106-108. doi:10.1097/QAD.0b013e3280118486

28. Gandhi M, Ameli N, Bacchetti P, et al. Atazanavir concentration in hair is the strongest predictor of outcomes on antiretroviral therapy. Clin Infect Dis. 2011;52(10):1267-1275. doi:10.1093/cid/cir131

29. van Zyl GU, van Mens TE, McIlleron H, et al. Low lopinavir plasma or hair concentrations explain second-line protease inhibitor failures in a resource-limited setting. J Acquir Immune Defic Syndr. 2011;56 (4):333-339. doi:10.1097/QAI.0b013e31820dc0cc

30. Baxi SM, Greenblatt RM, Bacchetti P, et al. Nevirapine concentration in hair samples is a strong predictor of virologic suppression in a prospective cohort of HIV-infected patients. PLoS One. 2015;10 (6):e0129100. doi:10.1371/journal.pone.0129100
31. Prasitsuebsai W, Kerr SJ, Truong KH, et al. Using lopinavir concentrations in hair samples to assess treatment outcomes on second-line regimens among Asian children. AIDS Res Hum Retroviruses. 2015;31(10):1009-1014. doi:10.1089/aid.2015.0111

32. Pintye J, Bacchetti P, Teeraananchai S, et al. Brief report: lopinavir hair concentrations are the strongest predictor of viremia in HIV-infected Asian children and adolescents on second-line antiretroviral therapy. J Acquir Immune Defic Syndr. 2017;76(4):367-371. doi:10.1097/QAI.0000000000001527

33. Murnane PM, Bacchetti P, Currier JS, et al. Tenofovir concentrations in hair strongly predict virologic suppression in breastfeeding women. AIDS. 2019;33(10):1657-1662. doi:10.1097/ QAD.0000000000002237

34. Gandhi M, Ameli N, Bacchetti P, et al. Protease inhibitor levels in hair strongly predict virologic response to treatment. AIDS. 2009;23 (4):471-478. doi:10.1097/QAD.0b013e328325a4a9

35. Yang X, Li X, Qiao S, Zhang Q, Shen Z, Zhou Y. Immunological and virologic outcomes of people living with HIV in Guangxi, China: 2012-2017. PLoS One. 2019;14(3):e0213205. doi:10.1371/journal. pone. 0213205

36. Zhang Q, Li X, Qiao S, Shen Z, Zhou Y. Comparing self-reported medication adherence measures with hair antiretroviral concentration among people living with HIV in Guangxi, China. AIDS Res Ther. 2020;17(1):8. doi:10.1186/s12981-020-00265-4

37. Zhang Q, Li X, Qiao S, Shen Z, Zhou Y. Factors influencing hair lamivudine concentration among people living with HIV in Guangxi, China. Antivir Ther. 2020;25(3):143-149. doi:10.3851/IMP3360

38. Cooper GA, Kronstrand R, Kintz P, Society of Hair T. Society of hair testing guidelines for drug testing in hair. Forensic Sci Int. 2012;218 (1-3):20-24. doi:10.1016/j.forsciint.2011.10.024

39. Sachs H. Quality control by the society of hair testing. Forensic Sci Int. 1997;84(1-3):145-150. doi:10.1016/S0379-0738(96)02057-9

40. Yang $\mathrm{H}, \mathrm{Chu} \mathrm{L}, \mathrm{Wu} \mathrm{Y}$, et al. LC-MS/MS quantification of nevirapine and its metabolites in hair for assessing long-term adherence. Molecules. 2020;25:23. doi:10.3390/molecules25235692

41. Eisinger RW, Dieffenbach CW, Fauci AS. HIV viral load and transmissibility of HIV infection: undetectable equals untransmittable. JAMA. 2019;321(5):451-452. doi:10.1001/jama.2018.21167

42. Feldman BJ, Fredericksen RJ, Crane PK, et al. Evaluation of the single-item self-rating adherence scale for use in routine clinical care of people living with HIV. AIDS Behav. 2013;17(1):307-318. doi:10.1007/s10461-012-0326-7

43. Koss CA, Natureeba P, Mwesigwa J, et al. Hair concentrations of antiretrovirals predict viral suppression in HIV-infected pregnant and breastfeeding Ugandan women. AIDS. 2015;29(7):825-830. doi:10.1097/QAD.0000000000000619

44. Chawana TD, Gandhi M, Nathoo K, et al. Defining a cutoff for atazanavir in hair samples associated with virological failure among adolescents failing second-line antiretroviral treatment. J Acquir Immune Defic Syndr. 2017;76(1):55-59. doi:10.1097/ QAI.0000000000001452

45. Cohan D, Natureeba P, Koss CA, et al. Efficacy and safety of lopinavir/ritonavir versus efavirenz-based antiretroviral therapy in HIV-infected pregnant Ugandan women. AIDS. 2015;29 (2):183-191. doi:10.1097/QAD.0000000000000531

46. Ngara B, Zvada S, Chawana TD, Stray-Pedersen B, Nhachi CFB, Rusakaniko S. A population pharmacokinetic model is beneficial in quantifying hair concentrations of ritonavir-boosted atazanavir: a study of HIV-infected Zimbabwean adolescents. BMC Pharmacol Toxicol. 2020;21(1):58. doi:10.1186/s40360-020-00437-y 


\section{Publish your work in this journal}

Patient Preference and Adherence is an international, peer-reviewed, open access journal that focusing on the growing importance of patient preference and adherence throughout the therapeutic continuum. Patient satisfaction, acceptability, quality of life, compliance, persistence and their role in developing new therapeutic modalities and compounds to optimize clinical outcomes for existing disease

states are major areas of interest for the journal. This journal has been accepted for indexing on PubMed Central. The manuscript management system is completely online and includes a very quick and fair peer-review system, which is all easy to use. Visit http:// www.dovepress.com/testimonials.php to read real quotes from published authors. 